PROCEEDINGS OF THE

AMERICAN MATHEMATICAL SOCIETY

Volume 140, Number 8, August 2012, Pages 2663-2670

S 0002-9939(2011)11130-4

Article electronically published on December 27, 2011

\title{
ARITHMETIC NORMAL FUNCTIONS AND FILTRATIONS ON CHOW GROUPS
}

\author{
JAMES D. LEWIS
}

(Communicated by Lev Borisov)

\begin{abstract}
Let $X / \mathbb{C}$ be a smooth projective variety, and let $\mathrm{CH}^{r}(X, m)$ be the higher Chow group defined by Bloch. Saito and Asakura defined a descending candidate Bloch-Beilinson filtration $\mathrm{CH}^{r}(X, m ; \mathbb{Q})=F^{0} \supset \cdots \supset$ $F^{r} \supset F^{r+1}=F^{r+2}=\cdots$, using the language of mixed Hodge modules. Another more geometrically defined filtration is constructed by Kerr and Lewis in terms of germs of normal functions. We show that under the assumptions (i) $X / \mathbb{C}=X_{0} \times \mathbb{C}$ where $X_{0}$ is defined over $\overline{\mathbb{Q}}$, and (ii) the general Hodge conjecture, that $F^{\bullet} \mathrm{CH}^{r}(X, m ; \mathbb{Q})$ coincides with the aforementioned geometric filtration. More specifically, it is characterized in terms of germs of reduced arithmetic normal functions.
\end{abstract}

\section{INTRODUCTION}

The notion of a (still conjectural) Bloch-Beilinson (B-B) filtration on Chow groups is an idea that originated with S. Bloch and was later fortified by Beilinson in terms of extension datum in a conjectural category of mixed motives. Over the years a number of candidate B-B filtrations have surfaced, due to S. Saito $\underline{\text { SS }}$, U. Jannsen, W. Raskind, J. Lewis Lew1, M. Kerr and J. Lewis K-L, P. Griffiths and M. Green, J. Murre, M. Saito and M. Asakura As, to name a few. The filtration in $[\mathrm{As}$ is also discussed in $[\mathrm{K}-\mathrm{L}$, where it also gives rise to an explicit geometrically defined filtration in terms of arithmetic normal functions. The natural question then is whether these two filtrations coincide. Two obvious consequences of an affirmative answer are that:

- The filtration in As can be described geometrically.

- The geometric filtration inherits the known properties of the one in $\mathrm{As}$, which makes use of the powerful cohomological machinery of mixed Hodge modules.

Our main result is the following:

Theorem 1.1. Let $X / \mathbb{C}$ be a smooth projective variety. Assume that (i) $X=$ $X_{0} \times \overline{\mathbb{Q}} \mathbb{C}$, where $X_{0}$ is defined over $\overline{\mathbb{Q}}$, and (ii) the general Hodge conjecture holds. Then the filtration in $\mathrm{As}$ admits a geometric description in terms of germs of (reduced) arithmetic normal functions.

For the convenience of the reader, we review the definition of the filtration in question and along the way prove more general results.

Received by the editors March 28, 2010 and, in revised form, March 16, 2011.

2010 Mathematics Subject Classification. Primary 14C25; Secondary 14C30, 14C35.

Key words and phrases. Bloch-Beilinson filtration, arithmetic normal function, Chow group.

The author was partially supported by a grant from the Natural Sciences and Engineering Research Council of Canada.

(C)2011 American Mathematical Society Reverts to public domain 28 years from publication 


\section{NOTATION AND CONVENTIONS}

(i) Throughout this paper we will assume that $k=\bar{k} \subset \mathbb{C}$ is an algebraically closed subfield and that $K / k$ (with $K \subset \mathbb{C}$ ) is a finitely generated field extension. Let $\mathcal{V}_{k}$ be the category of smooth projective varieties over $k$.

(ii) $\mathbb{Q}(r)=(2 \pi \mathrm{i})^{r} \mathbb{Q}$ (Tate twist).

(iii) For a $\mathbb{Q}$-mixed Hodge structure (MHS) $H=H_{\mathbb{Q}}$, we put

$$
\Gamma(H):=\operatorname{hom}_{\mathrm{MHS}}(\mathbb{Q}(0), H), \quad J(H):=\operatorname{Ext}_{\mathrm{MHS}}^{1}(\mathbb{Q}(0), H) .
$$

This is in the category of MHS which are not necessarily graded polarizable. Thus by [Ca, [Ja, Lemma 9.2],

$$
J(H)=\frac{W_{0} H_{\mathbb{C}}}{W_{0} H+F^{0} W_{0} H_{\mathbb{C}}} .
$$

(Note: With regard to the category of graded polarizable MHS, the corresponding $\operatorname{Ext}^{1}(\mathbb{Q}(0), H)$ is given by $W_{-1} H_{\mathbb{C}} /\left\{\left\{W_{0} H+F^{0} W_{0} H_{\mathbb{C}}\right\} \cap W_{-1} H_{\mathbb{C}}\right\} \hookrightarrow J(H)$, As, $\S 2.5]$. A formal consequence of the right exactness of $\operatorname{Ext}_{\mathrm{MHS}}^{1}(\mathbb{Q}(0),-)$ is that the higher extension groups vanish, viz., $\operatorname{Ext}_{\mathrm{MHS}}^{\nu}(\mathbb{Q}(0), H)=0$ for all $\nu \geq 2$, a fact established by Beilinson [Be, Cor. 1.10].)

(iv) Let $L \subset \mathbb{C}$ be any subfield and $X \in \mathcal{V}_{L}$. For singular cohomology we define $H^{i}(X, \mathbb{Q}):=H^{i}(X(\mathbb{C}), \mathbb{Q})$. The coniveau filtration is given by

$$
N_{L}^{\nu} H^{i}(X, \mathbb{Q}):=\operatorname{ker}\left(H^{i}(X, \mathbb{Q}) \rightarrow \lim _{Y \subset X / \overrightarrow{L, \mathrm{~cd}_{X}} Y \geq \nu} H^{i}(X \backslash Y, \mathbb{Q})\right) .
$$

(v) For $X \in \mathcal{V}_{\mathbb{C}}$, the general Hodge conjecture (abbreviated GHC) says that $N_{\mathbb{C}}^{\nu} H^{i}(X, \mathbb{Q})=N_{H}^{\nu} H^{i}(X, \mathbb{Q})$, where $N_{H}^{\nu} H^{i}(X, \mathbb{Q}):=$ largest sub-Hodge structure in $F^{\nu} H^{i}(X, \mathbb{C}) \cap H^{i}(X, \mathbb{Q})$.

(vi) $\mathrm{CH}^{r}\left(X_{K}, m\right)$ is the higher Chow group introduced in $\left[\mathrm{B}\right.$, and $\mathrm{CH}^{r}\left(X_{K}, m ; \mathbb{Q}\right)$ $:=\mathrm{CH}^{r}\left(X_{K}, m\right) \otimes \mathbb{Q}$.

Owing to the technical nature of this paper, the reader may find the following sources of background information useful. For a discussion of the general Hodge conjecture, see Lew2. Section 11 in Ja describes Beilinson's conjecture on a filtration on Chow groups for $X \in \mathcal{V}_{K}$. A good source of mixed Hodge theory is the survey article [Br-Z]. For extension classes of MHS, see [Ca and [Ja, §9]. For mixed Hodge modules, the reader should consult $\mathrm{As}$, and the references cited therein.

\section{A DESCENDING FILTRATION}

Consider fields $k \subset K \subset \mathbb{C}$, where $K / k$ is finitely generated. We consider the filtration constructed in [Lew1] in the case $m=0$, and more generally for $m \geq 0$ in As. We recall:

Theorem 3.1. Let $X \in \mathcal{V}_{K}$ be of dimension $d$. Then for all $r$, there is a filtration, depending on $k \subset K$,

$$
\begin{aligned}
C H^{r}\left(X_{K}, m ; \mathbb{Q}\right)= & F^{0} \supset F^{1} \supset \cdots \supset F^{\nu} \supset F^{\nu+1} \\
& \supset \cdots \supset F^{r} \supset F^{r+1}=F^{r+2}=\cdots,
\end{aligned}
$$

which satisfies the following:

(i) $F^{1}=C H_{\mathrm{hom}}^{r}\left(X_{K}, m ; \mathbb{Q}\right)$.

(ii) $F^{2} \subset \operatorname{ker} A J \otimes \mathbb{Q}: C H_{\mathrm{hom}}^{r}\left(X_{K} ;, m ; \mathbb{Q}\right) \rightarrow J\left(H^{2 r-m-1}\left(X_{K}(\mathbb{C}), \mathbb{Q}(r)\right)\right)$. 
(iii) $F^{\nu_{1}} C H^{r_{1}}\left(X, m_{1} ; \mathbb{Q}\right) \bullet F^{\nu_{2}} C H^{r_{2}}\left(X, m_{2} ; \mathbb{Q}\right) \subset F^{\nu_{1}+\nu_{2}} C H^{r_{1}+r_{2}}\left(X, m_{1}+m_{2} ; \mathbb{Q}\right)$, where $\bullet$ is the intersection product.

(iv) $F^{\nu}$ is preserved under the action of correspondences between smooth projective varieties in $\mathcal{V}_{K}$.

(v) Let $G r_{F}^{\nu}:=F^{\nu} / F^{\nu+1}$ and assume that the Künneth components of the diagonal class $\left.\left[\Delta_{X}\right]=\bigoplus_{p+q=2 d}\left[\Delta_{X}(p, q)\right] \in H^{2 d}(X \times X, \mathbb{Q}(d))\right)$ are algebraic and defined over $K$. Then

$$
\left.\Delta_{X}(2 d-2 r+\ell+m, 2 r-\ell-m)_{*}\right|_{G r_{F}^{\nu} C H^{r}(X, m ; \mathbb{Q})}=\delta_{\ell, \nu} \cdot \text { Identity. }
$$

(If we assume the conjecture that homological and numerical equivalence coincide, then (v) says that $G r_{F}^{\nu}$ factors through the Grothendieck motive.)

(vi) Let $D^{r}(X):=\bigcap_{\nu} F^{\nu}$ and $k=\overline{\mathbb{Q}}$. If the Bloch-Beilinson conjecture on the injectivity of the Abel-Jacobi map $(\otimes \mathbb{Q})$ holds for smooth quasi-projective varieties defined over $\overline{\mathbb{Q}}$ (see $\S 4$ of $[\mathrm{K}-\mathrm{L}]$ ), then $D^{r}(X)=0$.

It is instructive to briefly explain how this filtration comes about. For $X \in \mathcal{V}_{K}$, one can find $\mathcal{S} / k$ such that $k(\mathcal{S})$ is identified with $K$. One can then spread out $X / K$ to a family $\rho: \mathcal{X} \rightarrow \mathcal{S}$ (called a $k$-spread), where $\rho$ is a smooth and proper morphism of smooth quasi-projective varieties over $k$. Let $\eta$ be the generic point of $\mathcal{S}$, (hence $k(\mathcal{S})=k(\eta)$ and $K$ corresponds to an embedding $k(\eta) \stackrel{\approx}{\hookrightarrow} K \subset \mathbb{C}$ ), with $X_{K}$ identified with the generic fiber of $\rho$. Via M. Saito's notion of polarizable mixed Hodge modules (see $\mathrm{As}$, as well as $\S 4$ of $[\mathrm{K}-\mathrm{L}]$ ), there is a decreasing filtration $\mathcal{F}^{\nu} \mathrm{CH}^{r}(X / k, m ; \mathbb{Q})$, with the property that $\operatorname{Gr}_{\mathcal{F}}^{\nu} \mathrm{CH}^{r}(X / k, m ; \mathbb{Q}) \hookrightarrow E_{\infty}^{\nu, 2 r-\nu-m}(\rho)$, where $E_{\infty}^{\nu, 2 r-\nu-m}(\rho)$ is the $\nu$-th graded piece of a Leray filtration associated to $\rho$. More specifically, there is a cycle class map

$$
\mathrm{CH}^{r}(X, m ; \mathbb{Q}) \rightarrow \operatorname{Ext}_{\operatorname{MHM}(X)}^{2 r-m}\left(\mathbb{Q}_{X}(0), \mathbb{Q}_{X}(r)\right),
$$

where $\operatorname{MHM}(\mathcal{X})$ is the category of polarizable mixed Hodge modules on $\mathcal{X}$. There is the Leray spectral sequence associated to $\rho$ :

$$
\begin{gathered}
E_{2}^{p, q}=\operatorname{Ext}_{\operatorname{MHM}(\mathcal{S})}^{p}\left(\mathbb{Q}_{\mathcal{S}}(0), R^{q} \rho_{*} \mathbb{Q}_{X}(r)\right) \Rightarrow \operatorname{Ext}_{\operatorname{MHM}(X)}^{2 r-m}\left(\mathbb{Q}_{X}(0), \mathbb{Q}_{X}(r)\right), \\
p+q=2 r-m,
\end{gathered}
$$

which degenerates at $E_{2}$ since $\rho$ is smooth and proper. Then we put

$$
E_{\infty}^{\nu, 2 r-\nu-m}(\rho):=E_{2}^{\nu, 2 r-\nu-m}=\operatorname{Ext}_{\operatorname{MHM}(\mathcal{S})}^{\nu}\left(\mathbb{Q}_{\mathcal{S}}(0), R^{2 r-\nu-m} \rho_{*} \mathbb{Q}_{X}(r)\right) .
$$

Applying the Leray spectral sequence to the structure map $\mathcal{S} \rightarrow \operatorname{Spec}(k)$, together with $\operatorname{MHM}(\operatorname{Spec}(k))=$ MHS, we see that the term $E_{\infty}^{\nu, 2 r-\nu-m}(\rho)$ fits in a short exact sequence:

$$
0 \rightarrow \underline{E}_{\infty}^{\nu, 2 r-\nu-m}(\rho) \rightarrow E_{\infty}^{\nu, 2 r-\nu-m}(\rho) \rightarrow \underline{\underline{E}}_{\infty}^{\nu, 2 r-\nu-m}(\rho) \rightarrow 0,
$$

where

$$
\begin{aligned}
\underline{E}_{\infty}^{\nu, 2 r-\nu-m}(\rho) & =\Gamma\left(H^{\nu}\left(\mathcal{S}(\mathbb{C}), R^{2 r-\nu-m} \rho_{*} \mathbb{Q}(r)\right)\right) \\
\underline{E}_{\infty}^{\nu, 2 r-\nu-m}(\rho) & =\frac{J\left(W_{-1} H^{\nu-1}\left(\mathcal{S}(\mathbb{C}), R^{2 r-\nu-m} \rho_{*} \mathbb{Q}(r)\right)\right)}{\Gamma\left(G r_{W}^{0} H^{\nu-1}\left(\mathcal{S}(\mathbb{C}), R^{2 r-\nu-m} \rho_{*} \mathbb{Q}(r)\right)\right)} \\
& \subset J\left(H^{\nu-1}\left(\mathcal{S}(\mathbb{C}), R^{2 r-\nu-m} \rho_{*} \mathbb{Q}(r)\right)\right),
\end{aligned}
$$

\footnotetext{
${ }^{1}$ Not specifically stated in $\underline{\mathrm{As}}$, although expected to be true for the higher Chow groups.
} 
where the latter inclusion is a result of the short exact sequence

$$
\begin{gathered}
W_{-1} H^{\nu-1}\left(\mathcal{S}(\mathbb{C}), R^{2 r-\nu-m} \rho_{*} \mathbb{Q}(r)\right) \hookrightarrow W_{0} H^{\nu-1}\left(\mathcal{S}(\mathbb{C}), R^{2 r-\nu-m} \rho_{*} \mathbb{Q}(r)\right) \\
\rightarrow G r_{W}^{0} H^{\nu-1}\left(\mathcal{S}(\mathbb{C}), R^{2 r-\nu-m} \rho_{*} \mathbb{Q}(r)\right),
\end{gathered}
$$

and where the image

$$
\Gamma\left(G r_{W}^{0} H^{\nu-1}\left(\mathcal{S}(\mathbb{C}), R^{2 r-\nu-m} \rho_{*} \mathbb{Q}(r)\right)\right) \rightarrow J\left(W_{-1} H^{\nu-1}\left(\mathcal{S}(\mathbb{C}), R^{2 r-\nu-m} \rho_{*} \mathbb{Q}(r)\right)\right)
$$

is described as follows. Let $y \in \Gamma\left(G r_{W}^{0} H^{\nu-1}\left(\mathcal{S}(\mathbb{C}), R^{2 r-\nu-m} \rho_{*} \mathbb{Q}(r)\right)\right)$, and choose

$$
x \in W^{0} H^{\nu-1}\left(\mathcal{S}(\mathbb{C}), R^{2 r-\nu-m} \rho_{*} \mathbb{Q}(r)\right), x_{\mathbb{C}} \in F^{0} W^{0} H^{\nu-1}\left(\mathcal{S}(\mathbb{C}), R^{2 r-\nu-m} \rho_{*} \mathbb{C}\right),
$$

which map to $y$ under the surjection $W_{0} \rightarrow G r_{W}^{0}$. Then the image of $y$ in (2) is given by the image of $x-x_{\mathbb{C}}$ in $J\left(W_{-1} H^{\nu-1}\left(\mathcal{S}(\mathbb{C}), R^{2 r-\nu-m} \rho_{*} \mathbb{Q}(r)\right)\right)$. Under the identification of $K$ with $k(\eta)$, one then has (by definition)

$$
F^{\nu} \mathrm{CH}^{r}\left(X_{K}, m ; \mathbb{Q}\right)=\lim _{U \subset \overrightarrow{\mathcal{S}} / k} \mathcal{F}^{\nu} \mathrm{CH}^{r}\left(X_{U} / \overline{\mathbb{Q}}, m ; \mathbb{Q}\right), \quad X_{U}:=\rho^{-1}(U) .
$$

We set

$$
E_{\infty}^{\nu, 2 r-\nu-m}\left(\eta_{\mathcal{S}}\right)=\lim _{U \overrightarrow{\mathcal{C}} / k} E_{\infty}^{\nu, 2 r-\nu-m}(\rho)
$$

and the same definition for $\underline{E}_{\infty}^{\nu, 2 r-\nu-m}\left(\eta_{\mathcal{S}}\right)$ and $\underline{\underline{E}}_{\infty}^{\nu, 2 r-\nu-m}\left(\eta_{\mathcal{S}}\right)$. Specifically,

$$
\begin{gathered}
\underline{E}_{\infty}^{\nu, 2 r-\nu-m}\left(\eta_{\mathcal{S}}\right)=\Gamma\left(H^{\nu}\left(\eta_{\mathcal{S}}, R^{2 r-\nu-m} \rho_{*} \mathbb{Q}(r)\right)\right), \\
\underline{E}_{\infty}^{\nu, 2 r-\nu-m}\left(\eta_{\mathcal{S}}\right)=J\left(W_{-1} H^{\nu-1}\left(\eta_{\mathcal{S}}, R^{2 r-\nu-m} \rho_{*} \mathbb{Q}(r)\right)\right) / \Gamma\left(G r_{W}^{0}\right) .
\end{gathered}
$$

We have a short exact sequence,

$$
0 \rightarrow \underline{E}_{\infty}^{\nu, 2 r-\nu-m}\left(\eta_{\mathcal{S}}\right) \rightarrow E_{\infty}^{\nu, 2 r-\nu-m}\left(\eta_{\mathcal{S}}\right) \rightarrow \underline{\underline{E}}_{\infty}^{\nu, 2 r-\nu-m}\left(\eta_{\mathcal{S}}\right) \rightarrow 0,
$$

and an injection,

$$
\operatorname{Gr}_{F}^{\nu} \mathrm{CH}^{r}\left(X_{K}, m ; \mathbb{Q}\right) \hookrightarrow E_{\infty}^{\nu, 2 r-\nu-m}\left(\eta_{\mathcal{S}}\right) .
$$

(ii) We then define

$$
F^{\nu} \mathrm{CH}^{r}\left(X_{\mathbb{C}}, m ; \mathbb{Q}\right)=\lim _{k \subset \vec{K} \subset \mathbb{C}} F^{\nu} \mathrm{CH}^{r}\left(X_{K}, m ; \mathbb{Q}\right),
$$

which becomes a candidate B-B filtration on $\mathrm{CH}^{r}\left(X_{\mathbb{C}}, m ; \mathbb{Q}\right)$ in the case $k=\overline{\mathbb{Q}}$.

\section{Arithmetic normal FunCtions}

Let $X \in \mathcal{V}_{K}$ with $K / k$ finitely generated. We recall for the reader [K-L, $\S 4$ ] a geometric interpretation of the aforementioned descending filtration in terms of arithmetic normal functions, and prove some definitive results in the case where $X$ is obtained by base change from a corresponding variety in $\mathcal{V}_{k}$. So we consider $X \in \mathcal{V}_{K}$ with $k=\bar{k} \subset K$ and the paragraph following Theorem 3.1 above. Consider the coniveau subspace $N_{K}^{r-\nu+1} H^{2 r-\nu-m}(X, \mathbb{Q}(r)) \subset H^{2 r-\nu-m}(X, \mathbb{Q}(r))$. After possibly shrinking $\mathcal{S}, N_{K}^{r-\nu+1} H^{2 r-\nu-m}(X, \mathbb{Q}(r))$ determines a corresponding sub-VHS $N_{K}^{r-\nu+1} R^{2 r-\nu-m} \rho_{*} \mathbb{Q}(r) \subset R^{2 r-\nu} \rho_{*} \mathbb{Q}(r)$, which by semisimplicity considerations is a direct summand. Now let $Y \subset X / K$ be of (pure) codimension $r-\nu+1$ such that

$$
H_{Y}^{2 r-\nu-m}(X, \mathbb{Q}(r)) \rightarrow N_{K}^{r-\nu+1} H^{2 r-\nu-m}(X, \mathbb{Q}(r))
$$


is surjective, with desingularization $\tilde{Y} \rightarrow Y$ and composite map $\sigma: \tilde{Y} \rightarrow X$. Let us assume there is a $K$-cycle induced map

$$
\tilde{P}: H^{2 r-\nu-m}(X, \mathbb{Q}) \rightarrow H^{\nu-m-2}(\tilde{Y}, \mathbb{Q}),
$$

such that

$P:=\sigma_{*} \circ \tilde{P}: H^{2 r-\nu-m}(X, \mathbb{Q}(r)) \rightarrow N_{K}^{r-\nu+1} H^{2 r-\nu-m}(X, \mathbb{Q}) \subset H^{2 r-\nu-m}(X, \mathbb{Q}(r))$

is a projector.

Proposition 4.1. Given the above setting

(i) $P_{*} G r_{F}^{\nu} C H^{r}\left(X_{K}, m ; \mathbb{Q}\right)=0$.

(ii)

$$
\begin{aligned}
& \operatorname{Im}\left(G r_{F}^{\nu} C H^{r}\left(X_{K}, m ; \mathbb{Q}\right) \rightarrow \underline{\underline{E}}_{\infty}^{\nu, 2 r-\nu-m}\left(\eta_{\mathcal{S}}\right)\right) \\
& \quad \bigcap \Gamma\left(H^{\nu}\left(\eta_{\mathcal{S}}, N_{K}^{r-\nu+1} R^{2 r-\nu-m} \rho_{*} \mathbb{Q}(r)\right)\right)=0 .
\end{aligned}
$$

$$
\begin{aligned}
& \left\{G r_{F}^{\nu} C H^{r}\left(X_{K}, m ; \mathbb{Q}\right)\right. \\
& \left.\bigcap \operatorname{Im}\left(J\left(W_{-1} H^{\nu-1}\left(\eta_{\mathcal{S}}, N_{K}^{r-\nu+1} R^{2 r-\nu-m} \rho_{*} \mathbb{Q}(r)\right)\right) \rightarrow \underline{E}_{\infty}^{\nu, 2 r-\nu-m}\left(\eta_{\mathcal{S}}\right)\right)\right\}=0 .
\end{aligned}
$$

Proof. (i) For $[\xi] \in G r_{F}^{\nu} \mathrm{CH}^{r}\left(X_{K}, m\right.$; $\left.\mathbb{Q}\right)$, use the fact that $P_{*}([\xi])=\left[P_{*}(\xi)\right]=\left[\sigma_{*} \circ\right.$ $\left.\tilde{P}_{*}(\xi)\right] \in G r_{F}^{\nu} \mathrm{CH}^{r}\left(X_{K}, m ; \mathbb{Q}\right)$ factors through $\left[\tilde{P}_{*}(\xi)\right] \in G r_{F}^{\nu} \mathrm{CH}^{\nu-1}\left(\tilde{Y}_{K}, m ; \mathbb{Q}\right)=0$. (To help the reader out here, $\tilde{P}_{*}$ is induced by a cycle in $\mathrm{CH}^{d-r+\nu-1}\left(X_{K} \times \tilde{Y}_{K} ; \mathbb{Q}\right)$, and hence $\tilde{P}_{*} \mathrm{CH}^{r}\left(X_{K}, m ; \mathbb{Q}\right) \subset \mathrm{CH}^{\nu-1}\left(\tilde{Y}_{K}, m ; \mathbb{Q}\right)$. Now use the fact that $\tilde{P}_{*}$ is compatible with $G r_{F}^{\nu}$ and that $G r_{F}^{\nu} \mathrm{CH}^{\nu-1}\left(\tilde{Y}_{K}, m ; \mathbb{Q}\right)=0$, both statements due to Theorem 3.1.) For (ii) and (iii), observe that the respective intersections are preserved under $P_{*}$. Now apply (i).

Corollary 4.2. Let us assume the Hodge conjecture (hence with cycle induced $\tilde{P})$. Then $P_{*} G r_{F}^{\nu} C H^{r}\left(X_{\mathbb{C}}, m ; \mathbb{Q}\right)=0$.

Remark 4.3. (i) If $X \in \mathcal{V}_{k}$ where we recall $k=\bar{k}$, then we can arrange for $\tilde{P}$ to be induced by a cycle over $k$ by using a spread argument similar to that in the proof of Lemma 7.5 on page 1456 in L-S.

(ii) Corollary 4.2 is consistent with the conjectured equivalence of the two filtrations introduced and explained in [SS, Thm. (1-1)].

We now assume that $\mathcal{S}$ is affine. Let $V \subset \mathcal{S}(\mathbb{C})$ be a smooth closed affine subvariety of dimension $\nu-1$ with diagram of varieties, which we now view as defined over $\mathbb{C}$ :

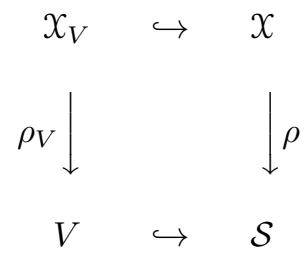


Correspondingly we have

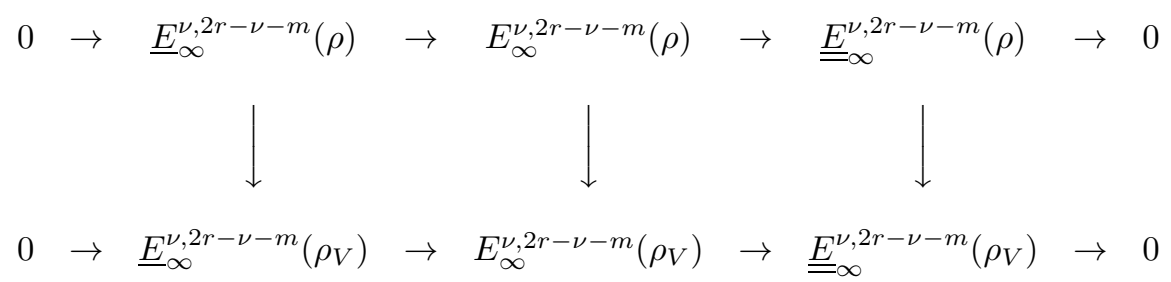

By the weak Lefschetz theorem for affine varieties (see, for example, [A, Lemma 2.5], $\underline{\underline{E}}^{\nu, 2 r-\nu-m}\left(\rho_{V}\right)=0$. Therefore a class $\xi \in \mathcal{F}^{\nu} \mathrm{CH}^{r}(X / k, m ; \mathbb{Q})$ determines a class $\lambda_{\xi}(V) \in \underline{E}_{\infty}^{\nu, 2 r-\nu-m}\left(\rho_{V}\right)$. Thinking of $V$ as part of a family $V=V_{0} \in\left\{V_{t}\right\}_{t \in W}$, we arrive at the notion of an arithmetic normal function $\lambda_{\xi}\left(V_{t}\right)$. To be more precise,

Definition 4.4 ([K-L, §4]). For $\xi \in \mathcal{F}^{\nu} \mathrm{CH}^{r}(X / k, m$; $\mathbb{Q})$, the corresponding $\lambda_{\xi}$ is called an arithmetic normal function.

The class of $[\xi] \in \underline{\underline{E}}_{\infty}^{\nu, 2 r-\nu-m}(\rho)$ is determined by $\lambda_{\xi}$ (topological invariant of $\lambda_{\xi}$; see $[\mathrm{K}-\mathrm{L}, \S 4])$. A reasonable expectation is that these arithmetic normal functions can be used to completely characterize $F^{\nu} \mathrm{CH}^{r}\left(X_{K}, m ; \mathbb{Q}\right)$. In the case where $X$ is a product (viz., where $X \in \mathcal{V}_{k}$ ), we obtain such an explicit description in Theorem4.6. To this end, consider the product situation with $X=S \times X$, all defined over $k$, with $K=k(S)$. Set

$$
H_{0}=W_{-1}\left(H^{\nu-1}\left(\eta_{S}, \mathbb{Q}\right) \otimes \frac{H^{2 r-\nu-m}(X, \mathbb{Q})}{N_{H}^{r-\nu+1} H^{2 r-\nu-m}(X, \mathbb{Q})}(r)\right),
$$

where $\eta_{S}$ is the generic point of $S / k$. A key ingredient due to mixed Hodge theory is the following:

Lemma 4.5. There is a natural map

$$
\underline{E}_{\infty}^{\nu, 2 r-\nu-m}\left(\eta_{S}\right) \rightarrow J\left(H_{0}\right) .
$$

Proof. We assume $S / k$ is affine and put

$$
\begin{gathered}
W_{j}=W_{j}\left(H^{\nu-1}(S, \mathbb{Q}) \otimes H^{2 r-\nu-m}(X, \mathbb{Q})(r)\right), \\
W_{j}^{H}=W_{j}\left(H^{\nu-1}(S, \mathbb{Q}) \otimes N_{H}^{r-\nu+1} H^{2 r-\nu-m}(X, \mathbb{Q})(r)\right) .
\end{gathered}
$$

The proof of the lemma boils down to showing that

$$
\Gamma\left(G r_{W}^{0}\right)=\Gamma\left(G r_{W^{H}}^{0}\right),
$$

together with the description of $\underline{E}_{\infty}^{\nu, 2 r-\nu-m}(\rho)$ in (1). We point out that a similar argument appears in [L-S, page 1485], but for completeness, we provide the details here. First of all, it is easier to "untwist" things by observing that $\Gamma\left(\mathrm{Gr}_{W}^{0}\right)$ can be identified with hom $\mathrm{MHS}\left(\mathbb{Q}(-r), V \otimes H^{2 r-\nu-m}(X, \mathbb{Q})\right)$, where $V=\operatorname{Gr}_{W}^{\nu+m} H^{\nu-1}(S, \mathbb{Q})$. But observe that the maximum weight of $H^{\nu-1}(S, \mathbb{Q})$ is $2 \nu-2$ (see [Ja, page 85]), and that $2 \nu-2 \geq \nu+m \Leftrightarrow \nu \geq m+2$. So we may assume that $\nu \geq m+2$. Further, an analysis of the MHS $H^{\nu-1}(S, \mathbb{Q})$ shows that $F^{\nu-1} V_{\mathbb{C}}=V_{\mathbb{C}}$. In particular this implies that hom $\operatorname{MHS}_{\mathrm{S}}\left(\mathbb{Q}(-r), V \otimes H^{2 r-\nu-m}(X, \mathbb{Q})\right) \subset V \otimes N_{H}^{r-\nu+1} H^{2 r-\nu-m}(X, \mathbb{Q})$; 
hence (3) follows. Finally, the lemma follows from the commutative diagram

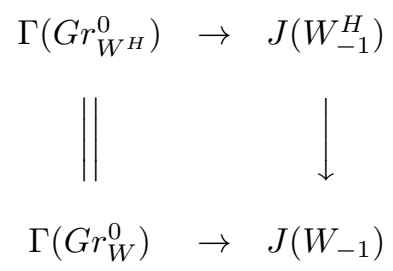

Now consider $\xi \in F^{\nu} \mathrm{CH}^{r}\left(X_{K}, m ; \mathbb{Q}\right)$. Such a class $\xi$ is represented by a corresponding class (still denoted by) $\xi \in \mathrm{CH}^{r}\left(S \times_{k} X, m ; \mathbb{Q}\right)$ for some affine $S$. Let $\lambda_{\xi}$ be the corresponding arithmetic normal function. More precisely, by $\lambda_{\xi}$, we mean the following. For smooth affine closed $V \subset S(\mathbb{C})$, we restrict ourselves to $\eta_{V}:=$ " $V \cap \eta_{S}$ " (with the obvious meaning), and where

$$
\lambda_{\xi}(V) \in \frac{J\left(W_{-1}\left[H^{\nu-1}\left(\eta_{V}, \mathbb{Q}\right) \otimes H^{2 r-\nu-m}(X, \mathbb{Q})(r)\right]\right)}{\Gamma\left(G r_{W}^{0}\left[H^{\nu-1}\left(\eta_{V}, \mathbb{Q}\right) \otimes H^{2 r-\nu-m}(X, \mathbb{Q})(r)\right]\right)} .
$$

The reduced arithmetic normal function $\underline{\lambda}_{\xi}$ has the characteristic property that its value lies in

$$
\underline{\lambda}_{\xi}(V) \in J\left(W_{-1}\left[H^{\nu-1}\left(\eta_{V}, \mathbb{Q}\right) \otimes \frac{H^{2 r-\nu-m}(X, \mathbb{Q})}{N_{H}^{r-\nu+1} H^{2 r-\nu-m}(X, \mathbb{Q})}(r)\right]\right),
$$

which via Lemma 4.5 is the image of $\lambda_{\xi}(V)$. We prove:

Theorem 4.6. In the case where $X \in \mathcal{V}_{k}$, and under the assumption of the $G H C$, the filtration $\left\{F^{\nu} \mathrm{CH}^{r}\left(X_{K}, m ; \mathbb{Q}\right)\right\}_{\nu \geq 0}$ is characterized by the germs of reduced arithmetic normal functions.

Proof. By Proposition 4.1 and under the assumption of the GHC, $\underline{\lambda}_{\xi}=0 \Leftrightarrow \lambda_{\xi}=0$. (This uses the fact that $N_{k}^{r-\nu+1} H^{2 r-\nu-m}(X, \mathbb{Q})=N_{H}^{r-\nu+1} H^{2 r-\nu-m}(X, \mathbb{Q})$ (since $k=\bar{k}$ ) and that $\left.\underline{\lambda}_{\xi}=0 \Rightarrow \lambda_{\xi}=\lambda_{P_{*} \xi}=0\right)$. But recall $\lambda_{\xi}=0 \Rightarrow[\xi]=0 \in$ $\underline{\underline{E}}_{\infty}^{\nu, 2 r-\nu-m}\left(\eta_{S}\right)$. Thus $[\xi] \in \underline{E}_{\infty}^{\nu, 2 r-\nu-m}\left(\eta_{S}\right)$, and its value is zero in $\underline{E}_{\infty}^{\nu, 2 r-\nu-m}\left(\eta_{S}\right)$ (equivalently, $\left.\xi=0 \in G r_{F}^{\nu} \mathrm{CH}^{r}\left(X_{K}, m ; \mathbb{Q}\right)\right)$ if and only if its value in $J\left(H_{0}\right)$ of Lemma 4.5 is zero, again using Proposition 4.1. Using the affine Lefschetz theorem (injectivity part of Lemma 2.5 in $\underline{\mathrm{A}}$, together with the fact that the MHS $H_{0}$ only involves negative weights), $\underline{\lambda}_{\xi}=0 \Rightarrow[\xi] \mapsto 0 \in J\left(H_{0}\right)$. (To be more specific, if $V_{1} \hookrightarrow V_{2}$ is an injection of MHS, then $J\left(W_{-1} V_{1}\right) \hookrightarrow J\left(W_{-1} V_{2}\right)$ is likewise an injection, the latter injection following from the short exact sequence $0 \rightarrow W_{-1} V_{1} \rightarrow$ $W_{-1} V_{2} \rightarrow W_{-1}\left(V_{2} / V_{1}\right) \rightarrow 0$, together with $\Gamma\left(W_{-1}\left(V_{2} / V_{1}\right)\right)=0$.) In summary, the image of $\xi \in F^{\nu} \mathrm{CH}^{r}\left(X_{K} ; \mathbb{Q}\right)$ in $E_{\infty}^{\nu, 2 r-\nu}\left(\eta_{S}\right)$ is zero $\Leftrightarrow \underline{\lambda}_{\xi}=0$; i.e., $\Leftrightarrow \xi=0 \in$ $\operatorname{Gr}_{F}^{\nu} \mathrm{CH}^{r}\left(X_{K}, m ; \mathbb{Q}\right)$, which is the same thing as saying $\Leftrightarrow \xi \in F^{\nu+1} \mathrm{CH}^{r}\left(X_{K}, m ; \mathbb{Q}\right)$.

Theorem 1.1 now follows immediately from this.

\section{ACKNOWLEDGEMENT}

The author is grateful to the referee for suggesting improvements in the presentation of this material. 


\section{REFERENCES}

[A] D. Arapura, The Leray spectral sequence is motivic, Inventiones Math. 160 (2005), 567589. MR2178703(2006m:14025)

[As] M. Asakura, Motives and algebraic de Rham cohomology, in "The Arithmetic and Geometry of Algebraic Cycles" (Banff), CRM Proc. Lect. Notes 24, AMS, 2000, 133-154. MR1736879 (2001c:14041)

[Be] A. Beilinson, Notes on absolute Hodge cohomology, Contemp. Math., Volume 55, Part I, Amer. Math. Soc. (1986), 35-68. MR862628 (87m:14019)

[Br-Z] J.-L. Brylinski and S. Zucker, An overview of recent advances in Hodge theory, in Several complex variables, VI, Encyclopedia Math. Sci. 69, Springer, Berlin (1990), 39-142. MR1095090 (91m:14010)

[B] S. Bloch, Algebraic cycles and higher K-theory, Advances in Math. 61 (1986), 267-304. MR852815 (88f:18010)

[Ca] J. Carlson, Extensions of mixed Hodge structures, Journées de geométrie algébrique d'Angers, 1979, Sijthoff \& Noordhof (1980), pp. 107-127. MR605338 (82g:14013)

[Ja] U. Jannsen, Mixed Motives and Algebraic K-Theory, Lecture Notes in Mathematics 1400, Springer-Verlag, Berlin (1990). MR1043451 (91g:14008)

[K-L] M. Kerr, J. D. Lewis, The Abel-Jacobi map for higher Chow groups II, Invent. Math. 170 (2) (2007), 355-420. MR2342640 (2008j:14005)

[Lew1] J. D. Lewis, A filtration on the Chow groups of a complex projective variety, Compositio Math. 128 (2001), 299-322. MR1858339 (2002h:14003)

[Lew2] J. D. Lewis, A survey of the Hodge conjecture, Second edition. Appendix B by B. Brent Gordon. CRM Monograph Series 10. American Mathematical Society, Providence, RI, 1999. MR1683216 (2000a:14010)

[L-S] J. D. Lewis, S. Saito, Algebraic cycles and Mumford-Griffiths invariants, Amer. J. Math. 129 (2007), no. 6, 1449-1499. MR2369886 (2008j:14014)

[SS] S. Saito, Motives and filtrations on Chow groups, II, in "The Arithmetic and Geometry of Algebraic Cycles", Proceedings of the CRM Summer School, June 7-19, 1998, Banff, Alberta, Canada (Editors: B. Gordon, J. Lewis, S. Müller-Stach, S. Saito and N. Yui), NATO Science Series 548 (2000), 321-346, Kluwer Academic Publishers. MR1744952 (2001d:14025)

Department of Mathematical and Statistical Sciences, 632 Central Academic Building, University of Alberta, Edmonton, Alberta T6G 2G1, Canada

E-mail address: lewisjd@ualberta.ca 